ERR A T UM

Stefan Wöhrer • Markus Raderer • Berthold Streubel •

Andreas Chott $\cdot$ Johannes Drach

\title{
Concomitant occurrence of MALT lymphoma and multiple myeloma
}

Published online: 15 July 2004

(C) Springer-Verlag 2004

\section{Ann Hematol (2004) DOI 10.1007/s00277-004 0870-y}

An oversight by the author led to the following error:

In the discussion section, paragraph 4 , line 1 it is stated that MALT lymphoma comprises $25-50 \%$ of all new lymphoma cases.
The online version of the original article can be found at http:// dx.doi.org/10.1007/s00277-004-0870-y

S. Wöhrer $(\bowtie) \cdot$ M. Raderer · J. Drach

Clinical Division of Oncology, Department of Medicine I,

University Hospital Vienna,

Währinger Gürtel 18-20, 1090 Vienna, Austria

e-mail: stefan.woehrer@meduniwien.ac.at

Tel.: +43-1-404004466

Fax: +43-1-404004451

B. Streubel · A. Chott

Department of Pathology,

University Hospital Vienna,

Währinger Gürtel 18-20, 1090 Vienna, Austria
This is wrong: Extranodal lymphomas comprise 25$50 \%$ of all new lymphomas but MALT lymphoma comprises only $8 \%$ of all new lymphoma cases. 